\title{
Community acquired COVID-19 related complications after coronary artery bypass grafting.
}

\author{
Eden Payabyab ${ }^{1}$, Ryan Chiu ${ }^{1}$, Miguel Armas ${ }^{2}$, Samuel Lang ${ }^{1}$, Dimitrios Avgerinos ${ }^{1}$, and \\ Charles Mack ${ }^{1}$ \\ ${ }^{1}$ Weill Cornell Medicine \\ ${ }^{2}$ New York-Presbyterian Queens
}

November 5, 2020

\begin{abstract}
Many hospitals will continue to see a surge in COVID-19 cases and halt of elective cases, while others pass the surge and begin to restart elective surgeries. Regardless of the phase each hospital encounters, the safety of both patients and staff must be taken into account.1,2 This case report describes a patient who underwent urgent coronary artery bypass grafting (CABG) at the beginning of the COVID-19 surge. The initial postoperative course was uneventful and the patient was discharged on postoperative day 4. The patient presented nearly two weeks later with a subsequent severe COVID-19 infection complicated by STEMI and stroke. This case not only demonstrates the risks and potential complications of caring for cardiac surgery patients during the COVID-19 pandemic, it is the only known reported case of a patient developing severe COVID-19 infection after a CABG resulting in STEMI and stroke due to hypercoagulable state associated with COVID-19.
\end{abstract}

Title: Community acquired COVID-19 related complications after coronary artery bypass grafting.

Authors: Eden C. Payabyab MD ${ }^{1}$, Ryan Chiu MD ${ }^{1}$, Miguel Armas $\mathrm{PA}^{2}$, Samuel J. Lang MD ${ }^{1}$, Dimitrios V. Avgerinos MD, $\mathrm{PhD}^{1}$, Charles A. Mack $\mathrm{MD}^{1}$

${ }^{1}$ Department of Cardiothoracic Surgery, Weill Cornell Medicine, New York, NY, USA

${ }^{2}$ New York Presbyterian Hospital-Queens, Flushing, N.Y.

Running title: COVID-19 STEMI after CABG

Address for correspondence :

Charles A Mack

525 East $68^{\text {th }}$ Street

Box 110

Suite M 104

New York, NY 10065

Phone (718) 670-2400

Email:cmack@med.cornell.edu

Fax (718) 661-7404

Funding: None 


\title{
Disclosures: None
}

Keywords: COVID-19, CABG, STEMI, hypercoagulable

Abbreviations: Coronary artery bypass graft (CABG), ST elevation myocardial infarction (STEMI), Coronavirus disease (COVID-19)

\begin{abstract}
Many hospitals will continue to see a surge in COVID-19 cases and halt of elective cases, while others pass the surge and begin to restart elective surgeries. Regardless of the phase each hospital encounters, the safety of both patients and staff must be taken into account. ${ }^{1,2}$ This case report describes a patient who underwent an urgent CABG at the beginning of the COVID-19 surge. The initial postoperative course was uneventful and the patient was discharged on postoperative day 4 . The patient presented nearly two weeks later with a subsequent severe COVID-19 infection complicated by STEMI and stroke. This case not only demonstrates the risks and potential complications of caring for cardiac surgery patients during the COVID-19 pandemic, it is the only known reported case of a patient developing severe COVID-19 infection after a CABG resulting in STEMI and stroke due to hypercoagulable state associated with COVID-19.
\end{abstract}

\section{CASE REPORT}

Informed consent was obtained from the patient for publication of this case report and any accompanying images and was approved by the New York Presbyterian-Queens Institutional Review Board.

A 60 year-old male with hypertension, hyperlipidemia, non-insulin dependent diabetes, initially with unstable angina and associated dyspnea. Catheterization revealed severe triple vessel coronary artery disease. The following day he underwent an urgent three-vessel CABG (left internal mammary to left anterior descending, and separate saphenous vein grafts to posterior descending and first circumflex marginal). The postoperative echocardiogram showed normal ventricular function. His postoperative course was uncomplicated and he was discharged on postoperative day 4 . Thirteen days following discharge he presented the emergency department with 4 days of progressive shortness of breath associated with a productive cough and intermittent blood tinged sputum. He denied any chest pain on presentation. His vital signs were significant for mild tachycardia and hypoxia requiring a $100 \%$ nonrebreather mask to obtain a saturation of $97 \%$. Admission electrocardiogram (EKG) showed anterior and inferior ischemia with ST elevations (Figure 1). Pertinent laboratory blood work showed initial troponin-t of $0.537 \mathrm{ng} / \mathrm{ml}$ with a peak of $4.1 \mathrm{ng} / \mathrm{ml}$, a D-Dimer of 11 , $344 \mathrm{ng} / \mathrm{ml}$, and a C-reactive protein $5.55 \mathrm{mg} / \mathrm{dl}$. A chest radiograph revealed bilateral pulmonary infiltrates typically seen with COVID-19 (Figure 2). His presentation to the ED occurred at the very beginning of the COVID-19 surge. Given his community risk of exposure to COVID-19 in Queens, NY, a SARS-CoV-2 RT PCR was performed and detected the presence of the virus. A transthoracic echocardiogram demonstrated new severe left ventricular global hypokinesis with akinesis in the basal inferior and inferolateral segments. The estimated ejection fraction was $20 \%$.

He was started on intravenous heparin and dual antiplatelet therapy (with aspirin and clopidogrel) for the STEMI for high suspicion of a COVID-19 associated hypercoagulable state. Given the patient's hemodynamic stability, absence of chest pain, and scarcity of resources during the initial COVID-19 surge, a cardiac catheterization was deferred. During the early period of the initial COVID-19 surge, his treatment included a five-day course of hydroxychloroquine and azithromycin with close monitoring for hypoxia with supplemental oxygen as needed.

The patient's subsequent hospital course over the next week was complicated by ventricular tachycardia requiring electrical cardioversion and subsequent paroxysmal atrial fibrillation. The patient continued treatment with heparin anticoagulation and dual antiplatelet therapy. Despite therapeutic anticoagulation his hospital course was further complicated by emboli to his toes and a left hemiparesis. Computerized axial tomography and magnetic resonance imaging of the brain were consistent with acute embolic infarcts involving both cerebellar hemispheres, right thalamus, left temporal lobe and both occipital/frontal and parietal lobes. A repeat echocardiogram was performed which ruled out both apical and left atrial thrombus. 
Repeat imaging showed no hemorrhagic conversion of the stroke allowing for transition to oral anticoagulation for his atrial fibrillation. The patient continued to require supplemental oxygenation and hospital admission. He remained asymptomatic from his STEMI, with residual left sided motor weakness. He continued to work with physical and speech therapy and was discharged to a skilled nursing facility on hospital day 45 . Two months following discharge he was noted to have made a near full recovery. He is ambulatory without assistance. Transthoracic echocardiogram demonstrated an ejection fraction of $40 \%$ with inferior hypokinesis.

\section{DISCUSSION}

Whether the initial surge in COVID-19 patients has subsided and hospitals expand their operative caseloads or there is a subsequent increase in COVID-19 cases, there will inevitably be some degree of COVID transmission within healthcare setting and community. Surgeons should be cognizant of the prevalence of COVID-19 in their community and institution, and include it in their differential should postoperative complications arise $^{3,4,5}$ In our case report, the patient recovered from an uncomplicated CABG but experienced severe sequelae of COVID-19 disease.

On presentation to the emergency department, the patient was found to have a STEMI. Given his high risk of exposure to COVID-19 and absence of chest pain at the peak of the pandemic he was treated medically. Other stricken localities have experienced similar decision making processes. ${ }^{6}$

The patient was found to have elevated d-dimers, a marker of a hypercoagulable state, which is consistent with reports of severe hypercoagulability seen in COVID-19. ${ }^{7,8}$ While there have been reports of patients contracting COVID-19 after undergoing CABG, our case demonstrates the first report of thrombotic complications due to COVID-19 in a patient after CABG. ${ }^{4}$

Our patient's hypercoagulable state may have predisposed him to graft thrombosis leading to a STEMI. Other causes of STEMI have been reported in patients with COVID-19 including direct viral entry through the angiotensin-converting enzyme 2 receptor leading to cellular toxicity, hypoxic related myocyte injury and immune-mediated cytokine storm. ${ }^{9}$ His presentation with a STEMI is likely to be multifactorial, however without a catheterization we are unable to confirm if this was due to graft/native vessel thrombosis or direct myocyte injury.

Our patient's hospital course was complicated by other events that may be attributed to the hypercoagulable state associated with COVID-19. He experienced an acute embolic stroke with residual left hemiparesis and blues toes thought to be due to microthrombi. The events occurred despite being fully anticoagulated with heparin. Possible sources of the emboli are the brief episode of atrial fibrillation or the formation of thrombi in the hypokinetic left ventricle in combination with hypercoagulable diathesis.

As hospitals begin to transition back to an increased operative case load, surgeons will have to be aware of both community and hospital acquired COVID-19 infection. In addition, they should be cognizant of the potential thrombotic sequelae of COVID-19 in patients undergoing CABG. With relation to cardiac surgery outcome and quality reporting, COVID-19 may affect publicly reported patient outcomes and may require new risk adjustments.

Figure 1 


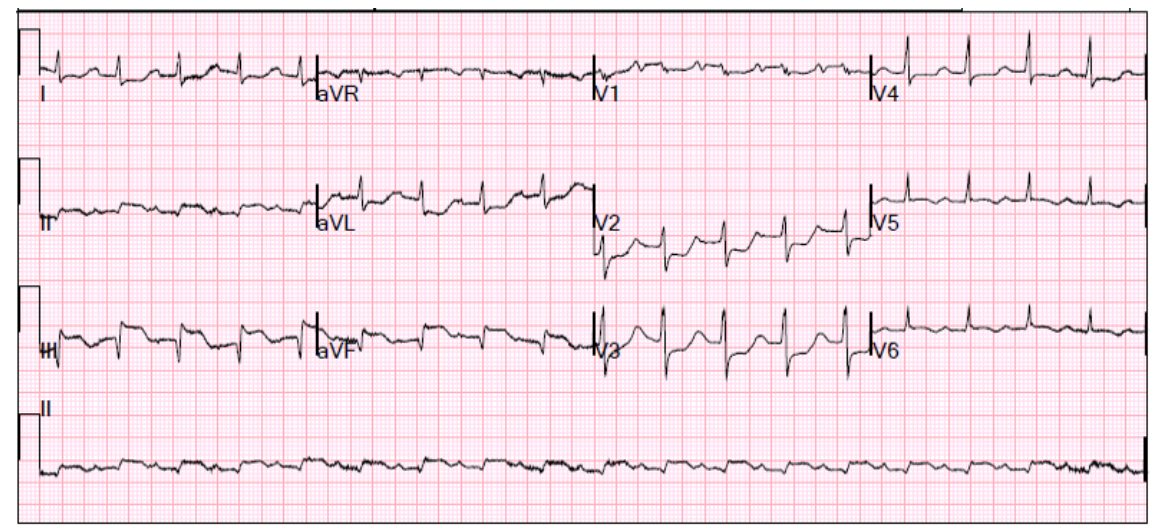

Admission EKG showing inferior lead ST elevation and anterior/septal ST depression

\section{Figure 2}

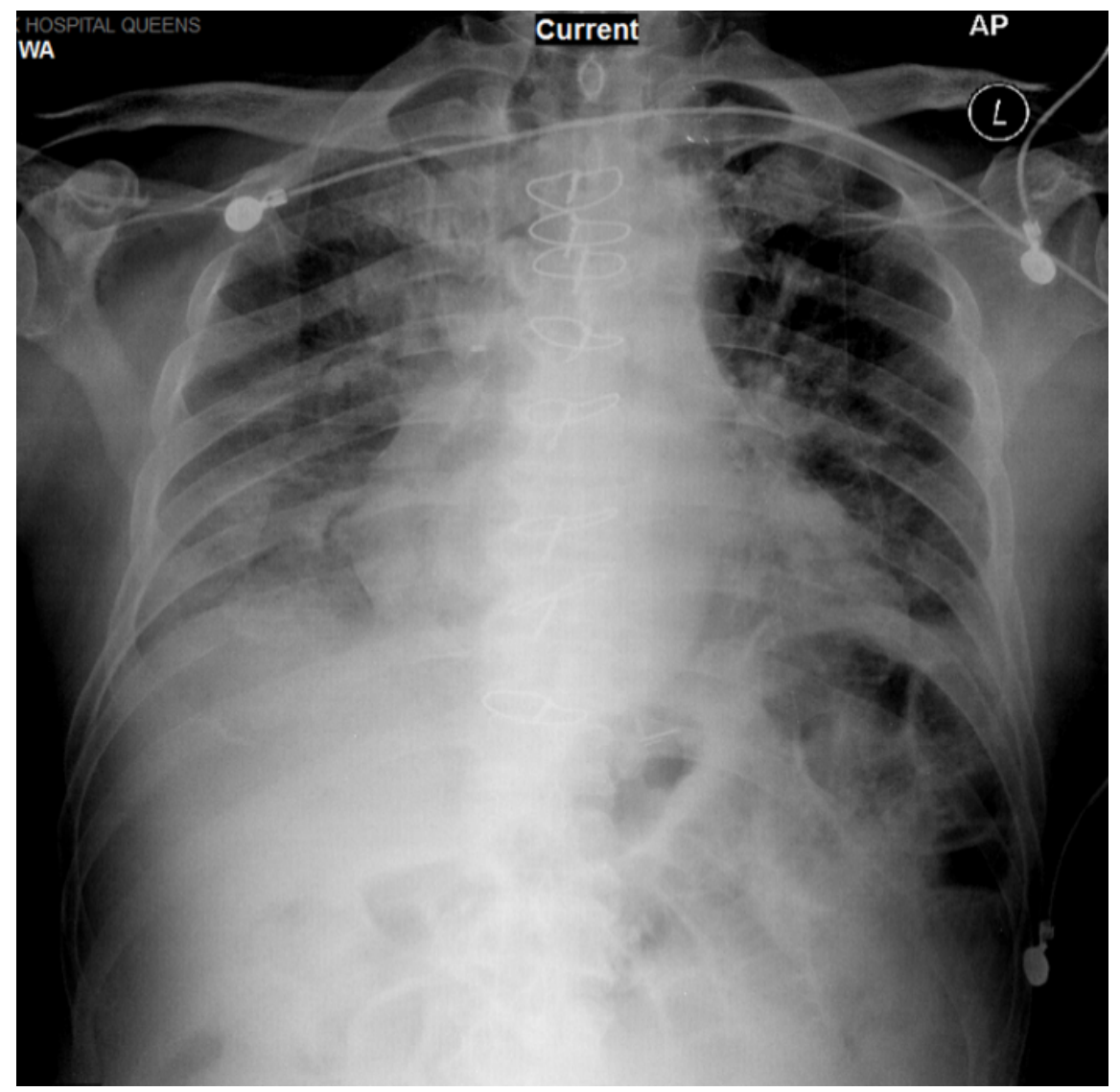

Chest radiograph demonstrating bilateral pulmonary infiltrates typically seen with COVID-19

\section{References:}

1. Prachand VN, Milner R, Angelos P, et al. Medically necessary, time-sensitive procedures: scoring system to ethically and efficiently manage resource scarcity and provider risk during the COVID-19 pandemic. $J$ Am 
Coll Surg . 2020. Aug;231(2):281-288

2. Farrington WJ, Mack CA, Karas MG, Ivascu NS, Robinson NB, Iannacone E, Lau C, Mick SL, Girardi LN. New York's perspective of COVID-19: Effect and impact on cardiac surgery. J Card Surg. 2020 Sept 16; doi $10.1111 /$ jocs. 15043

3. Stahel PF. How to risk-stratify elective surgery during the COVID-19 pandemic? Patient Saf Surg . 2020. Mar 31;14:8.doi:10.1186/s13037-020-00235-9

4. Fisher B, Seese L, Ibrahim S, Kilic A. The importance of repeat testing in detecting the coronavirus disease 2019 (COVID-19) in a coronary artery bypass grafting patient. J Card Surg. 2020 Jun;35(6)1342-1344. doi: $10.1111 /$ jocs. 14604 .

5. Lei S, Jiang F, Su W, et al. Clinical characteristics and outcomes of patients undergoing surgeries during the incubation period of COVID-19 infection. EClinicalMedicine . 2020 Apr 5;21:100331. doi: 10.1016/j.eclinm.2020.100331.

6. Zeng J, Huang J, Pan L. How to balance acute myocardial infarction and COVID-19: the protocols from Sichuan Provincial People's Hospital.Intensive Care Med . 2020 Jun;46(6):1111-1113. doi: 10.1007/s00134020-05993-9.

7. Klok FA, Kruip MJHA, van der Meer NJM, et al. Incidence of thrombotic complications in critically ill ICU patients with COVID-19. Thromb Res . 2020 Jul;191:145-147. doi: 10.1016/j.thromres.2020.04.013.

8. Spiezia L, Boscolo A, Poletto F, et al. COVID-19-related severe hypercoagulability in patients admitted to intensive care unit for acute respiratory failure. Thromb Haemost . 2020 Jun;50(1):223-226. doi:10.1007/s11239-020-02160-1

9. Zhu H, Rhee J-W, Cheng P, et al. Cardiovascular complications in patients with COVID-19: consequences of viral toxicities and host immune response. Curr Cardiol Rep . 2020 Apr 21;22(5):32. doi: 10.1007/s11886020-01292-3

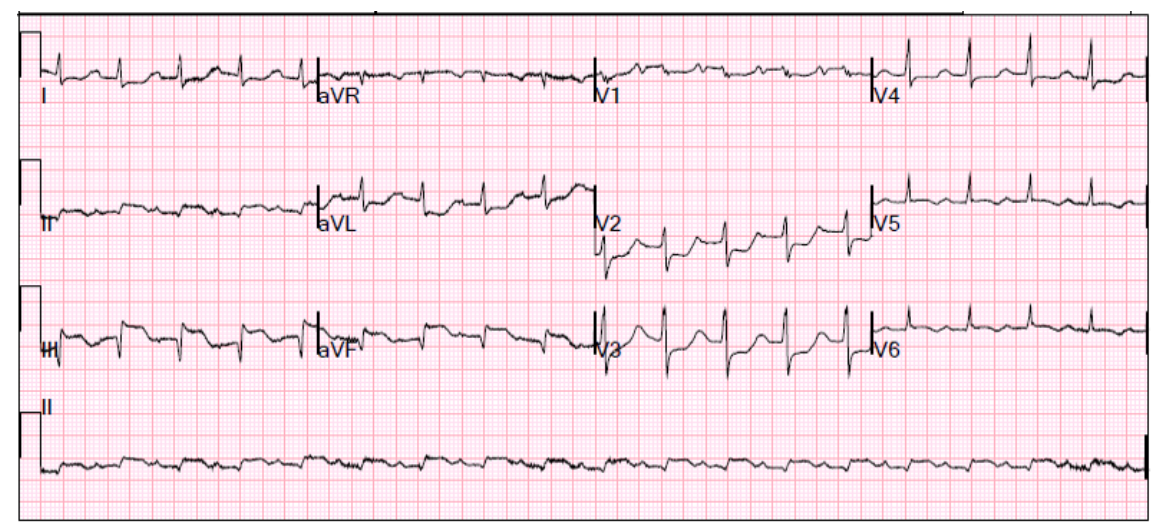




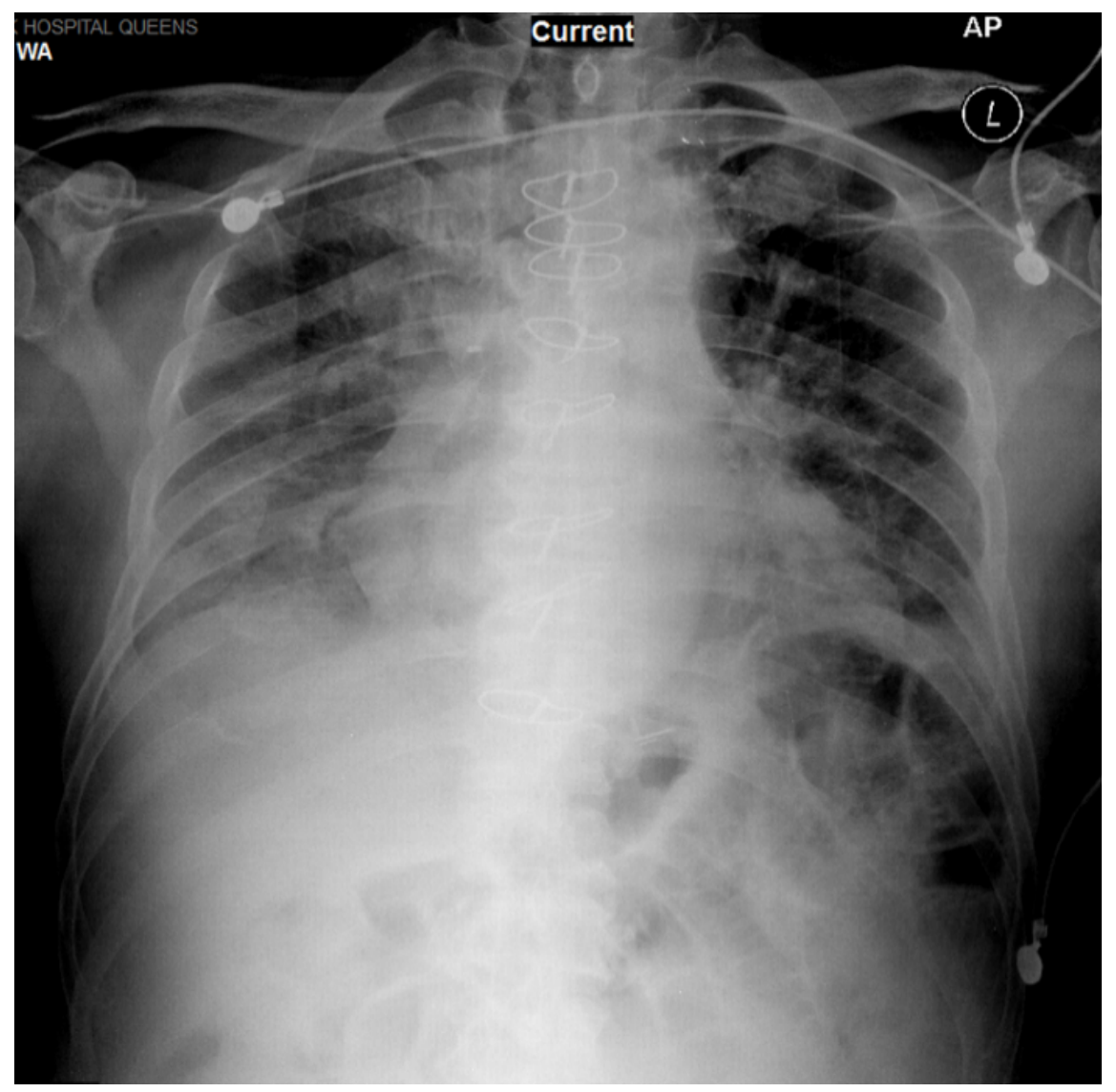

